hep-th/9908007

\title{
Compactification of Type IIB String Theory on Calabi-Yau Threefolds
}

\author{
Robert Böhm ${ }^{a}$, Holger Günther ${ }^{a}$, Carl Herrmann ${ }^{b}$, Jan Louis $^{a}$ 中 \\ ${ }^{a}$ Martin-Luther-Universität Halle-Wittenberg, \\ FB Physik, D-06099 Halle, Germany \\ ${ }^{b}$ Centre de Physique Théorique, CNRS - Luminy Case 90\%, \\ F-13288 Marseille Cedex 9, France
}

\begin{abstract}
We study compactifications of type IIB supergravity on Calabi-Yau threefolds. The resulting low energy effective Lagrangian is displayed in the large volume limit and its symmetry properties - with specific emphasis on the $S L(2, \mathbf{Z})$ - are discussed. The explicit map to type IIA string theory compactified on a mirror Calabi-Yau is derived. We argue that strong coupling effects on the worldsheet break the $S L(2, \mathbf{Z})$.
\end{abstract}

August 1999

\footnotetext{
${ }^{1}$ email: robert@hera.physik.uni-halle.de, guenther@hera.physik.uni-halle.de, herrmann@cptsu5.univmrs.fr, j.louis@physik.uni-halle.de.
} 


\section{Introduction}

Compactifications of string theories on 6-dimensional Calabi-Yau manifolds result in string vacua with four flat Minkowskian dimensions $(d=4)$ and, depending on the type of Calabi-Yau manifold, a fixed number of preserved supercharges. Specifically, string vacua with $N=2$ supersymmetry in $d=4$ are obtained by compactifying type II strings on Calabi-Yau threefolds $Y_{3}$, the heterotic string on $K 3 \times T^{2}$ or the type I string on $K 3 \times T^{2}$. It is believed that all of the resulting string vacua are perturbative descriptions of different regions in one and the same moduli space [1, 2]. In this paper we confine our attention to the region of the moduli space where type II string compactifications are the appropriate perturbative theories.

In $d=10$ type II string theories come in two different versions, the non-chiral type IIA and the chiral type IIB string theory but upon compactification on Calabi-Yau threefolds they are related by mirror symmetry. More precisely, type IIB compactified on $Y_{3}$ is

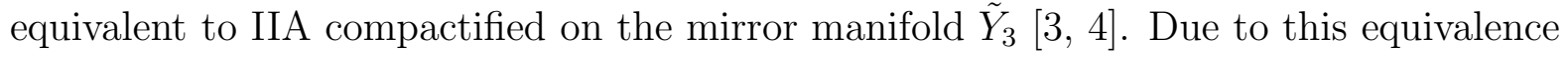
one has the choice to study either compactification but since there is no simple covariant action of type IIB supergravity in $d=10$ [5] type IIA compactifications appear to be easier [6]. In terms of the underlying conformal field theory the two theories in $d=4$ only differ by the GSO-projection and thus can be discussed on the same footing [3, 4]. However, it is believed that the type IIB theory in $d=10$ has an exact non-perturbative $S L(2, \mathbf{Z})$ symmetry [7] which is not shared by type IIA (in $d=10$ ). Thus, it is of interest to study the 'fate' of this $S L(2, \mathbf{Z})$ symmetry upon compactification to $d=4$ [8] and this is one of the goals of this paper. For that reason we focus on geometrical compactifications (and not the conformal field theory versions) of the effective type IIB theory for most parts of this paper. In addition, the general type IIB effective Lagrangian and the explicit map to the type IIA vacua has only been given in a special case [9, 10] and as a byproduct we close this gap.

This paper is organized as follows. In section 2 we recall some of the properties of type IIB supergravity in $d=10$. In section 3 we discuss the compactification on Calabi-Yau threefolds and display the four-dimensional low energy effective action in the large volume limit. Apart from the gravitational multiplet one finds $h_{(1,1)}$ tensor multiplets, $h_{(1,2)}$ vector multiplets and one "double-tensor" multiplet. We concentrate on the couplings of the tensor multiplets since the couplings of the vector multiplets have been given before [11, 4, 12]. In addition the $S L(2, \mathbf{Z})$ - inherited from the $d=10$ theory - acts on the tensor multiplets while the vector multiplets are left invariant. In section 4 the explicit map between type IIB compactified on $Y_{3}$ and type IIA compactified on the mirror manifold $\tilde{Y}_{3}$ is displayed. This map enables us to also discuss the geometry of the tensor multiplets in terms of a holomorphic prepotential and obtain the worldsheet instanton corrections. We find that the $S L(2, \mathbf{Z})$ is manifest at the string tree level in the large volume limit of the compactification but broken once strong coupling effects on the worldsheet (worldsheet instantons) are taken into account. Furthermore, the $S L(2, \mathbf{Z})$ is also present in the large complex structure limit of type IIA vacua but rather obscure in the standard field variables. Section 5 contains our conclusions and some of the technical aspects of this paper are collected in an appendix. 


\section{Type IIB Supergravity in $d=10$}

The bosonic massless modes of the type IIB string are the graviton $g_{M N}(M, N=$ $0, \ldots, 9)$, a doublet of antisymmetric tensors $B_{M N}^{I}(I=1,2)$, two real scalar fields $\phi, l$ and a 4-form $\mathcal{D}_{M N P Q}$ with a self-dual field strength. $g_{M N}, B_{M N}^{1}$ and $\phi$ arise in the NS-NS sector while $B_{M N}^{2}, l$ and $\mathcal{D}_{M N P Q}$ reside in the $\mathrm{R}$-R sector; $\phi$ is the dilaton of type IIB string theory.

The difficulty of constructing a covariant low energy effective action for these massless modes is due to the presence of the self-dual 5 -form field strength. The field equations of type IIB supergravity on the other hand are well known [5]. However, if one does not impose the self-duality condition a covariant action (in the Einstein frame) can be given 《13⿻

$$
\begin{aligned}
S=- & \frac{1}{2} \int d^{10} x \sqrt{-g}\left(\mathcal{R}-\frac{1}{4} \operatorname{Tr}\left(\partial M \partial M^{-1}\right)+\frac{3}{4} H^{I} M_{I J} H^{J}\right. \\
& \left.+\frac{5}{6} F^{2}+\frac{1}{96 \sqrt{-g}} \epsilon_{I J} \mathcal{D} \wedge H^{I} \wedge H^{J}\right)
\end{aligned}
$$

where $\mathcal{R}$ is the scalar curvature and we abbreviated

$$
\begin{aligned}
M_{I J} & \equiv \frac{1}{\operatorname{Im} \lambda}\left(\begin{array}{cc}
|\lambda|^{2} & -\operatorname{Re} \lambda \\
-\operatorname{Re} \lambda & 1
\end{array}\right), \quad \lambda \equiv l+i e^{-\phi}, \\
H_{M N P}^{I} & \equiv \partial_{[M} B_{N P]}^{I}=\frac{1}{3}\left(\partial_{M} B_{N P}^{I}+\partial_{N} B_{P M}^{I}+\partial_{P} B_{M N}^{I}\right) \\
F_{M N P Q R} & \equiv \partial_{[M} \mathcal{D}_{N P Q R]}+\frac{3}{4} \epsilon_{I J} B_{[M N}^{I} \partial_{P} B_{Q R]}^{J} .
\end{aligned}
$$

In eq. (1) we suppress space-time indices; in particular, the topological term $\mathcal{D} \wedge H^{I} \wedge H^{J}$ is contracted with the ten-dimensional $\epsilon$-tensor while all other terms are contracted with the ten-dimensional metric. Finally, we choose $\epsilon_{12}=+1$. The type IIB supergravity is obtained as the Euler-Lagrange equations of the action (1) together with the self-duality condition

$$
F_{M N P Q R}=\frac{1}{5 ! \sqrt{-g}} \epsilon_{M N P Q R S T U V W} F^{S T U V W} .
$$

This theory has a number of symmetries. First of all there are the gauge transformations of the 4 -form (with parameters $\Sigma_{N P Q}$ )

$$
\delta \mathcal{D}_{M N P Q}=\partial_{[M} \Sigma_{N P Q]},
$$

under which all other fields are invariant. Secondly, there are the gauge transformations of the two antisymmetric tensors (with parameters $\Omega_{N}^{I}$ ) which also transform the 4-form

$$
\begin{aligned}
\delta B_{M N}^{I} & =\partial_{[M} \Omega_{N]}^{I} \\
\delta \mathcal{D}_{M N P Q} & =-\frac{3}{4} \epsilon_{I J} \Omega_{[M}^{I} H_{N P Q]}^{J}
\end{aligned}
$$

\footnotetext{
${ }^{2}$ Recently a covariant action including the self-dual 4-form has been constructed [14 but here we choose to follow the procedure outlined in refs. 13] for compactifying type IIB supergravity and use (1) instead.
} 
Finally, there is an $S L(2, \mathbf{Z})$ acting as follows

$$
\begin{aligned}
\lambda & \mapsto \lambda^{\prime}=\frac{a \lambda+b}{c \lambda+d}, \\
H_{M N P}^{I} & \mapsto H_{M N P}^{\prime I}=\Lambda_{J}^{I} H_{M N P}^{J},
\end{aligned}
$$

where

$$
\Lambda=\left(\begin{array}{cc}
d & c \\
b & a
\end{array}\right), \quad a d-b c=1, \quad a, b, c, d \in \mathbf{Z} .
$$

Using (2) one shows that the matrix $M$ transforms according to

$$
M \mapsto M^{\prime}=\Lambda^{-1 T} M \Lambda^{-1}
$$

\section{Calabi-Yau Compactifications of Type IIB Super- gravity}

\subsection{The Spectrum}

Let us now study the compactification of the type IIB low energy effective theory on Calabi-Yau threefolds $Y_{3}$. The resulting theory in $d=4$ has $N=2$ supersymmetry and the low energy spectrum comes in appropriate $N=2$ supermultiplets. More specifically one finds that the 10-dimensional metric $g_{M N}$ decomposes into the 4-dimensional metric $g_{\mu \nu}(\mu, \nu=0, \ldots, 3), 2 \times h_{(1,2)}$ deformations of the complex structure $\delta g_{\alpha \beta}, \delta g_{\bar{\alpha} \bar{\beta}}(\alpha, \beta=$ $1,2,3)$ and $h_{(1,1)}$ deformations of the Kähler class $\delta g_{\alpha \bar{\beta}}$. The Hodge numbers $h_{(1,1)}\left(h_{(1,2)}\right)$ count the harmonic $(1,1)$-forms $((1,2)$-forms $)$ on $Y_{3}$. The antisymmetric tensors $B_{M N}^{I}$ decompose into a doublet of antisymmetric tensors $B_{\mu \nu}^{I}$ and $2 \times h_{(1,1)}$ scalar modes $B_{\alpha \bar{\beta}}^{I}$. Finally the 4-form decomposes into $h_{(1,1)}$ (real) antisymmetric tensors $\mathcal{D}_{\mu \nu \alpha \bar{\beta}}$ and $h_{(1,2)}+1$

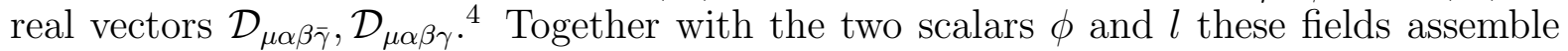
into the following $N=2$ supermultiplets:

$$
\begin{array}{ll}
\text { gravitational multiplet } & \left(g_{\mu \nu}, \mathcal{D}_{\mu \alpha \beta \gamma}\right), \\
\text { double - tensor multiplet } & \left(B_{\mu \nu}^{I}, \phi, l\right), \\
h_{(1,2)} \text { vector multiplets } & \left(\mathcal{D}_{\mu \alpha \beta \bar{\gamma}}, \delta g_{\alpha \beta}, \delta g_{\bar{\alpha} \bar{\beta}),},\right. \\
h_{(1,1)} \text { tensor multiplets } & \left(\mathcal{D}_{\mu \nu \alpha \bar{\beta}}, \delta g_{\alpha \bar{\beta}}, B_{\alpha \bar{\beta}}^{I}\right),
\end{array}
$$

where we only display the bosonic components. The gravitational multiplet contains 2 gravitini while all the other multiplets contain two Weyl fermions.f In $d=4$ an antisymmetric tensor describes one physical degree of freedom and thus can always be dualized to a scalar. At the level of supermultiplets this duality relates both the tensor and the double-tensor multiplet to a hypermultiplet which contains four real scalar degrees of

\footnotetext{
${ }^{3}$ In fact at the classical level one has the larger group $S L(2, \mathbf{R})$ which is broken by quantum corrections to $S L(2, \mathbf{Z})$.

${ }^{4}$ Note that the dimensionally reduced self-duality condition (3) relates $\mathcal{D}_{\alpha \beta \bar{\alpha} \bar{\beta}}$ to $\mathcal{D}_{\mu \nu \gamma \bar{\gamma}}, \mathcal{D}_{\mu \alpha \beta \gamma}$ to $\mathcal{D}_{\mu \bar{\alpha} \bar{\beta} \bar{\gamma}}$ and $\mathcal{D}_{\mu \alpha \beta \bar{\gamma}}$ to $\mathcal{D}_{\mu \bar{\alpha} \bar{\beta} \gamma}$.

${ }^{5}$ The double-tensor multiplet has not been constructed as an off-shell multiplet but we expect that it exists since it appears in the low energy limit of type IIB string theory.
} 
freedom. In this dual basis the low energy spectrum features $h_{(1,1)}+1$ hypermultiplets and $h_{(1,2)}$ vector multiplets apart from the gravitational multiplet.

The couplings of the vector multiplets have been obtained before [11, 14, 12] and thus we exclusively focus on the couplings of the $h_{(1,1)}$ tensor and the universal double-tensor multiplet. (Or in other words we consider Calabi-Yau threefolds with $h_{(1,2)}=0$.) On a Calabi-Yau threefold the Kähler deformations of the metric can be expanded in terms of harmonic $(1,1)$-forms $\omega_{\alpha \bar{\beta}}^{a}$ according to

$$
\begin{array}{rlrl}
\delta g_{\alpha \bar{\beta}} & =\hat{v}^{a}(x) \omega_{\alpha \bar{\beta}}^{a}, & B_{\alpha \bar{\beta}}^{I}=\hat{b}^{I a}(x) \omega_{\alpha \bar{\beta}}^{a}, \\
\mathcal{D}_{\mu \nu \alpha \bar{\beta}}=\hat{D}_{\mu \nu}^{a}(x) \omega_{\alpha \bar{\beta}}^{a}, & a=1, \ldots, h_{(1,1)} .
\end{array}
$$

In terms of these variables the tensor multiplets consist of $\left(\hat{D}_{\mu \nu}^{a}, \hat{v}^{a}, \hat{b}^{I a}\right)$.

Before we display the four-dimensional Lagrangian let us collect a few more properties of Calabi-Yau threefolds [15, 16]. The Kähler form $J$ is defined as

$$
J=i \delta g_{\alpha \bar{\beta}} d \xi^{\alpha} \wedge d \bar{\xi}^{\bar{\beta}}
$$

where the $\xi^{\alpha}$ are the complex coordinates on $Y_{3}$. The volume $V$ can be expressed in terms of $J$ according to

$$
V=i \int \sqrt{g} d^{6} \xi=\frac{1}{6} \int J \wedge J \wedge J
$$

On the space of $(1,1)$-forms one defines a metric

$$
G_{a b}=-\frac{i}{V} \int \omega_{\alpha \bar{\beta}}^{a} \omega_{\gamma \bar{\delta}}^{b} g^{\alpha \bar{\delta}} g^{\gamma \bar{\beta}} \sqrt{g} d^{6} \xi
$$

The intersection numbers of $Y_{3}$ are given by

$$
\kappa_{a b c}=\int \omega^{a} \wedge \omega^{b} \wedge \omega^{c}
$$

Both, the metric and the volume, can be expressed in terms of the moduli $\hat{v}^{a}$ and the intersection numbers

$$
\begin{aligned}
V & =\frac{1}{6} \kappa_{a b c} \hat{v}^{a} \hat{v}^{b} \hat{v}^{c} \\
G_{a b} & =-V^{-1} \kappa_{a b c} \hat{v}^{c}+\frac{1}{4} V^{-2} \kappa_{a c d} \hat{v}^{c} \hat{v}^{d} \kappa_{b e f} \hat{v}^{e} \hat{v}^{f} .
\end{aligned}
$$

\subsection{The Lagrangian}

The next step is the construction of the dimensionally reduced type IIB low energy effective theory. We follow the procedure outlined in refs. [13] and reduce the 10-dimensional action (回) imposing the self-duality condition on the field equations by hand. The resulting field equations in $d=4$ can be shown to be the Euler-Lagrange equations of a 
4-dimensional action. The technical details of the reduction procedure are deferred to the appendix and we only give the final result

$$
\begin{aligned}
\sqrt{-g}^{-1} \mathcal{L}= & -\frac{1}{2} \mathcal{R}-\frac{1}{4} e^{2 \phi_{4}} V(v)\left(\partial_{\mu} l\right)^{2}-\left(\partial_{\mu} \phi_{4}\right)^{2}-\frac{1}{6} e^{-3 \phi_{4}} V^{\frac{1}{2}}(v) H_{\mu \nu \rho}^{I} M_{I J} H^{J \mu \nu \rho} \\
& -G_{a b}(v) \partial_{\mu} v^{a} \partial^{\mu} v^{b}-e^{\phi_{4}} V^{\frac{1}{2}}(v) G_{a b}(v) \partial_{\mu} b^{I a} M_{I J} \partial^{\mu} b^{J b} \\
& -\frac{2}{3} e^{-2 \phi_{4}} V(v) G_{a b}(v)\left(F_{\mu \nu \rho}^{a}+\epsilon_{I J} H_{\mu \nu \rho}^{J} b^{I a}\right)\left(F^{b \mu \nu \rho}+\epsilon_{I J} H^{J \mu \nu \rho} b^{I b}\right) \\
& -\frac{i}{12 \sqrt{-g}} \epsilon^{\mu \nu \rho \sigma} \kappa_{a b c} \epsilon_{I J}\left(F_{\mu \nu \rho}^{a} b^{I b} \partial_{\sigma} b^{J c}-\frac{2}{3} \epsilon_{K L} H_{\mu \nu \rho}^{I} b^{J a} b^{K b} \partial_{\sigma} b^{L c}\right)
\end{aligned}
$$

where we redefined the field variables compared to the expansion parameters of eqs. (9) according to

$$
D_{\mu \nu}^{a} \equiv \hat{D}_{\mu \nu}^{a}+\frac{1}{2} \epsilon_{I J} B_{\mu \nu}^{I} b^{J a}, \quad F_{\mu \nu \rho}^{a} \equiv \partial_{[\mu} D_{\nu \rho]}^{a}, \quad b^{I a} \equiv \frac{2}{3} \hat{b}^{I a} .
$$

Furthermore, the kinetic terms for the $(1,1)$-moduli $\hat{v}^{a}$ and the dilaton decoupled due to the definition of the four-dimensional dilaton 12

$$
e^{-2 \phi_{4}}=V(v) e^{-2 \phi},
$$

and 'rotated' moduli fields

$$
v^{a}=\hat{v}^{a} e^{\phi / 2} .
$$

Note that (14) and (15) are only valid in the limit where the 10-dimensional theory is a good approximation, i.e. when $V$ is large. This limit is termed the 'large volume limit' but generically subleading $\left(\alpha^{\prime}\right)$ corrections are also important. These are briefly discussed in section 4.3 .

As we already discussed an antisymmetric tensor in four spacetime dimensions is dual to a scalar and so we can dualize the $h_{(1,1)}+2$ antisymmetric tensor fields $D_{\mu \nu}^{a}$, $B_{\mu \nu}^{I}$ in the action (15). This is done most conveniently by adding $h_{(1,1)}+2$ Lagrange multipliers $g_{a}, h_{I}$ to the Lagrangian

$$
\mathcal{L}^{\prime}=\mathcal{L}+i \epsilon^{\mu \nu \rho \sigma} H_{\mu \nu \rho}^{I} \partial_{\sigma} h_{I}+i \epsilon^{\mu \nu \rho \sigma} F_{\mu \nu \rho}^{a} \partial_{\sigma} g_{a} .
$$

The (algebraic) equations of motion of $\mathcal{L}^{\prime}$ for $F_{\mu \nu \rho}^{a}$ and $H_{\mu \nu \rho}^{I}$ can then be used to eliminate these fields in favour of the scalars $g_{a}$ and $h_{I}$. The resulting Lagrangian reads

$$
\begin{aligned}
\sqrt{-g}^{-1} \mathcal{L}^{\prime}= & -\frac{1}{2} \mathcal{R}-\frac{1}{4} e^{2 \phi_{4}} V\left(\partial_{\mu} l\right)^{2}-\left(\partial_{\mu} \phi_{4}\right)^{2}-G_{a b} \partial_{\mu} b^{1 a} \partial^{\mu} b^{1 b} \\
& -G_{a b} \partial_{\mu} v^{a} \partial^{\mu} v^{b}-e^{2 \phi_{4}} V G_{a b}\left(\partial_{\mu} b^{2 a}-l \partial_{\mu} b^{1 a}\right)\left(\partial^{\mu} b^{2 b}-l \partial^{\mu} b^{1 b}\right) \\
& -\frac{e^{2 \phi_{4}}}{16 V}\left(G^{-1}\right)^{a b}\left(\partial_{\mu} g_{a}-\frac{1}{2} \kappa_{a c d} \epsilon_{I J} b^{I c} \partial_{\mu} b^{J d}\right)\left(\partial^{\mu} g_{b}-\frac{1}{2} \kappa_{b e f} \epsilon_{K L} b^{K e} \partial^{\mu} b^{L f}\right) \\
& -\frac{1}{4} e^{2 \phi_{4}} V^{-1}\left(\partial_{\mu} h_{2}-b^{1 a}\left(\partial_{\mu} g_{a}-\frac{1}{6} \kappa_{a b c} \epsilon_{I J} b^{I b} \partial_{\mu} b^{J c}\right)\right)^{2} \\
& -\frac{1}{4} e^{4 \phi_{4}}\left(\partial_{\mu} h_{1}+l \partial_{\mu} h_{2}-\left(l b^{1 a}-b^{2 a}\right)\left(\partial_{\mu} g_{a}-\frac{1}{6} \kappa_{a b c} \epsilon_{I J} b^{I b} \partial_{\mu} b^{J c}\right)\right)^{2}
\end{aligned}
$$

where in addition the $\phi_{4}$-dependence is displayed more explicitly. 


\subsection{Symmetry Properties}

The symmetries of the 10-dimensional theory discussed in section 2 are also manifest in $d=4$. The action (15) is invariant under $h_{(1,1)}+2$ gauge transformations (with parameters $\Sigma_{\nu}^{a}, \Omega_{\nu}^{I}$ ) of the antisymmetric tensors

$$
\delta D_{\mu \nu}^{a}=\partial_{[\mu} \Sigma_{\nu]}^{a}, \quad \delta B_{\mu \nu}^{I}=\partial_{[\mu} \Omega_{\nu]}^{I} .
$$

In addition we have $2 \times h_{(1,1)}$ continuous Peccei-Quinn (PQ) symmetries (with parameters $\left.c^{I a}\right)$ acting on the scalar fields $b^{I a}$ and the antisymmetric tensors $D_{\mu \nu}^{a}$

$$
\delta b^{I a}=c^{I a}, \quad \delta D_{\mu \nu}^{a}=-\epsilon_{I J} c^{I a} B_{\mu \nu}^{J} .
$$

These symmetries are 'inherited' from the ten-dimensional symmetries (41), (5). Note that in the dual basis the gauge transformations (21) manifest themselves as $h_{(1,1)}+2$ additional continuous PQ symmetries (with parameters $\tilde{c}_{a}, \hat{c}_{I}$ )

$$
\delta g_{a}=\tilde{c}_{a}, \quad \delta h_{I}=\hat{c}_{I}
$$

while (22) transmogrifies into

$$
\delta b^{I a}=c^{I a}, \quad \delta g_{a}=\frac{1}{2} \kappa_{a b c} \epsilon_{I J} c^{I b} b^{J c}, \quad \delta h_{I}=-\epsilon_{I J}\left(c^{J a} g_{a}+\frac{1}{6} \kappa_{a b c} \epsilon_{K L} b^{J a} c^{K b} b^{L c}\right) .
$$

One of the distinct features of the type IIB theory in $d=10$ is its $S L(2, \mathbf{Z})$ invariance (6)-(8). This symmetry is of importance since it includes a strong-weak coupling duality as one of its generators. Thus it is of interest to study the fate of this symmetry in the $d=4$ action. Since eq. (15) was obtained as a straight dimensional reduction of the action (11) the $S L(2, \mathbf{Z})$ will also be manifest in (15) but due to the field redefinitions (17), (18) it also acts on the Calabi-Yau moduli $v^{a}$ and altogether becomes more involved. More precisely, using (6)-(8), (17) and (18), one finds

$$
v^{a} \mapsto v^{a}|c \lambda+d|, \quad e^{-2 \phi_{4}} \mapsto \frac{e^{-2 \phi_{4}}}{|c \lambda+d|}, \quad l \mapsto \frac{a c|\lambda|^{2}+(b d+a d) l+b d}{|c \lambda+d|^{2}},
$$

where $\lambda=l+i V^{-\frac{1}{2}} e^{-\phi_{4}}$. The antisymmetric tensors $B_{\mu \nu}^{I}$ and the scalars $b^{I a}$ inherit the transformation law (6) and obey

$$
\begin{aligned}
B_{\mu \nu}^{I} & \mapsto \Lambda_{J}^{I} B_{\mu \nu}^{J}, \\
b^{I a} & \mapsto \Lambda_{J}^{I} b^{J a}
\end{aligned}
$$

while the $D_{\mu \nu}^{a}$ remain invariant. From (19) one infers that the dual scalars $g_{a}$ are invariant whereas the $h_{I}$ transform with the inverse matrix

$$
h_{I} \mapsto \Lambda_{I}^{-1 J} h_{J}
$$

It can be explicitly checked that the Lagrangian (15) is invariant under the transformations specified in (25) and (26) and thus at the string tree level and in the large volume limit the $S L(2, \mathbf{Z})$ is present in $d=4$ [8]. 


\section{The Map between IIA and IIB}

\subsection{Preliminaries}

Type IIA string theory compactified on Calabi-Yau threefolds features $h_{(1,1)}$ vector multiplets, $h_{(1,2)}$ hypermultiplets and one tensor multiplet in its low energy spectrum. The tensor multiplet can be dualized to an additional hypermultiplet. A type IIB compactification on $Y_{3}$ is equivalent to a type IIA compactification on the mirror manifold $\tilde{Y}_{3}$ once the appropriate (worldsheet) quantum corrections are taken into account. This equivalence is a property of the underlying conformal field theory [17] but has also been demonstrated for the geometrical compactifications on Calabi-Yau manifolds [18. On the mirror manifold the Hodge numbers are reversed and one has

$$
h_{(1,1)}\left(Y_{3}\right)=h_{(1,2)}\left(\tilde{Y}_{3}\right), \quad h_{(1,2)}\left(Y_{3}\right)=h_{(1,1)}\left(\tilde{Y}_{3}\right) .
$$

The low energy spectrum of the two theories is most easily compared in the dual basis where the $h_{(1,1)}+1$ hypermultiplets of IIB are identified with the $h_{(1,2)}+1$ hypermultiplets of type IIA. The purpose of this section is to explicitly display the map between the field variables of type IIB and type IIA. For $h_{(1,1)}\left(Y_{3}\right)=1$ this map was given in ref. [9].

Let us start by recalling the low energy effective action of type IIA. The universal hypermultiplet of IIA which is the dual of the tensor multiplet contains the dilaton $\phi_{\mathrm{A}}$, the dual $\tilde{\phi}$ of an antisymmetric tensor and two scalars $\zeta^{0}, \tilde{\zeta}^{0}$ from the R-R sector. The scalars of the remaining $h_{(1,2)}$ hypermultiplets are denoted by $z^{a}, \bar{z}^{\bar{a}}, \zeta^{a}, \tilde{\zeta}^{a}$ where $a=1, \ldots, h_{(1,2)}$. Đ The $z^{a}, \bar{z}^{\bar{a}}$ arise from the NS-NS sector while the $\zeta^{a}, \tilde{\zeta}^{a}$ come from the R-R sector. The tree level Lagrangian for these hypermultiplets is known to be [19, 12]

$$
\begin{aligned}
\sqrt{-g}^{-1} \mathcal{L}= & -\frac{1}{2} \mathcal{R}-\left(\partial_{\mu} \phi_{\mathrm{A}}\right)^{2}-G_{a b}(z, \bar{z}) \partial^{\mu} z^{a} \partial_{\mu} \bar{z}^{\bar{b}} \\
& -\frac{1}{4} e^{4 \phi_{\mathrm{A}}}\left(\partial_{\mu} \tilde{\phi}+\zeta^{i} \partial_{\mu} \tilde{\zeta}_{i}-\tilde{\zeta}^{i} \partial_{\mu} \zeta_{i}\right)^{2}+\frac{1}{2} e^{2 \phi_{\mathrm{A}}} R_{i j}(z, \bar{z}) \partial^{\mu} \zeta^{i} \partial_{\mu} \zeta^{j} \\
& +\frac{1}{2} e^{2 \phi_{\mathrm{A}}} R^{-1 i j}(z, \bar{z})\left(I_{i k}(z, \bar{z}) \partial^{\mu} \zeta^{k}+\partial^{\mu} \tilde{\zeta}_{i}\right)\left(I_{j l}(z, \bar{z}) \partial_{\mu} \zeta^{l}+\partial_{\mu} \tilde{\zeta}_{j}\right)
\end{aligned}
$$

where $i, j, k, l=0, \ldots, h_{(1,2)}$. The scalar manifold spanned by the $4 \times\left(h_{(1,2)}+1\right)$ scalars is constrained by $N=2$ supergravity to be a quaternionic manifold [20] and this has been explicitly verified for (29) in ref. [19]. However, this scalar manifold is not the most general quaternionic manifold but at the string tree level further constrained by the cmap [4]. That is, the submanifold spanned by the deformations of the complex structure $z^{a}$ has to be a special Kähler manifold [11. I More precisely, the metric $G_{a b}(z, \bar{z})$ is Kähler and furthermore determined by a holomorphic prepotential $F(X)$ which is a homogenous function of degree two $\left(F(\lambda X)=\lambda^{2} F(X)\right)$. Specifically, one has

$$
G_{a b}=\frac{\partial}{\partial z^{a}} \frac{\partial}{\partial \bar{z}^{\bar{b}}} K
$$

\footnotetext{
${ }^{6}$ Here we use a slightly inconsistent notation since we reserved the index $a$ to label $(1,1)$-forms. However, we are anticipating the mirror map between type IIA and type IIB which identifies the $(1,1)$ forms on $Y_{3}$ with the $(1,2)$-forms on the mirror manifold $\tilde{Y}_{3}$ and for that reason we use the same indices already at this stage.

${ }^{7}$ The reason is that in type IIB vacua the $(1,2)$-forms reside in vector multiplets and the geometry of their scalars is constrained to be special Kähler.
} 
where

$$
\begin{aligned}
K & =-\log \left[X^{i} \bar{F}_{i}(\bar{X})+\bar{X}^{i} F_{i}(X)\right], \\
F_{i}(X) & \equiv \frac{\partial F}{\partial X^{i}}, \quad z^{i}=\frac{X^{i}}{X^{0}} \quad\left(z^{0}=1\right) .
\end{aligned}
$$

Furthermore, the matrices $R$ and $I$ in (29) are also determined by the prepotential according to

$$
\begin{aligned}
R_{i j} & =\operatorname{Re} \mathcal{N}_{i j}, \quad I_{i j}=\operatorname{Im} \mathcal{N}_{i j}, \\
\mathcal{N}_{i j} & =\frac{1}{4} \bar{F}_{i j}-\frac{(N z)_{i}(z N)_{j}}{(z N z)}
\end{aligned}
$$

where

$$
\begin{array}{rlrl}
F_{i j} & =\frac{\partial^{2} F}{\partial X^{i} \partial X^{j}}, \quad N_{i j} & =\frac{1}{4}\left(F_{i j}+\bar{F}_{i j}\right), \\
(N z)_{i} & =N_{i j} z^{j}, \quad(z N z)=z^{i} N_{i j} z^{j} .
\end{array}
$$

\subsection{The Map}

The geometrical compactification of the effective low energy IIB supergravity from $d=10$ to $d=4$ is only valid in the large volume limit of $Y_{3}$. In this limit the Calabi-Yau volume $V$ is a cubic function of the $(1,1)$ moduli $v^{a}$ and the intersection numbers $\kappa_{a b c}$ are constant. In order to display the map between IIA and IIB one has to take a similar limit on the IIA side. This limit - termed the 'large complex structure limit' - has been studied in the literature [18, 21]. For us the important point is that such a limit exists and in this limit the prepotential of the complex structure moduli is given by

$$
F=\frac{i}{3 !} \kappa_{a b c} \frac{X^{a} X^{b} X^{c}}{X^{0}} \equiv\left(X^{0}\right)^{2} f(z), \quad f(z)=\frac{i}{3 !} \kappa_{a b c} z^{a} z^{b} z^{c} .
$$

Using (30), (31), (34) and $z^{a}=x^{a}+i y^{a}$ one obtains the Kähler potential and the metric

$$
\begin{aligned}
K & =-\ln (\kappa y y y), \\
G_{a b}(y) & =-\frac{3}{2}\left(\frac{(\kappa y)_{a b}}{(\kappa y y y)}-\frac{3}{2} \frac{(\kappa y y)_{a}(\kappa y y)_{b}}{(\kappa y y y)^{2}}\right),
\end{aligned}
$$

where we abbreviated

$$
(\kappa y y y)=\kappa_{a b c} y^{a} y^{b} y^{c}, \quad(\kappa y y)_{a}=\kappa_{a b c} y^{b} y^{c}, \quad(\kappa y)_{a b}=\kappa_{a b c} y^{c} .
$$

The matrices defined in (32) simplify considerably and are given by

$$
\begin{aligned}
I & =\frac{1}{4}\left(\begin{array}{cc}
-\frac{1}{3}(\kappa x x x) & \frac{1}{2}(\kappa x x)_{b} \\
\frac{1}{2}(\kappa x x)_{a} & -(\kappa x)_{a b}
\end{array}\right), \\
R & =-\frac{1}{24}(\kappa y y y)\left(\begin{array}{cc}
1-4 G_{a b} x^{a} x^{b} & 4 G_{a b} x^{b} \\
4 G_{a b} x^{a} & -4 G_{a b}
\end{array}\right), \\
R^{-1} & =-24(\kappa y y y)^{-1}\left(\begin{array}{cc}
1 & x^{b} \\
x^{a} & -\frac{1}{4} G^{-1 a b}+x^{a} x^{b}
\end{array}\right) .
\end{aligned}
$$


Inserting (37) into (29) yields

$$
\begin{aligned}
\sqrt{-g}^{-1} \mathcal{L}= & -\frac{1}{2} \mathcal{R}-\left(\partial_{\mu} \phi_{\mathrm{A}}\right)^{2}-G_{a b} \partial_{\mu} y^{a} \partial^{\mu} y^{b}-G_{a b} \partial_{\mu} x^{a} \partial^{\mu} x^{b} \\
& -\frac{1}{8} e^{2 \phi_{\mathrm{A}}} V\left(\partial_{\mu} \zeta_{0}\right)^{2}-\frac{1}{2} e^{2 \phi_{\mathrm{A}}} V G_{a b}\left(x^{a} \partial_{\mu} \zeta_{0}-\partial_{\mu} \zeta^{a}\right)\left(x^{b} \partial^{\mu} \zeta_{0}-\partial^{\mu} \zeta^{b}\right) \\
& -\frac{1}{2} e^{2 \phi_{\mathrm{A}}} V^{-1} G^{-1 a b}\left(\partial_{\mu} \tilde{\zeta}_{a}+\frac{1}{8}(\kappa x x)_{a} \partial_{\mu} \zeta_{0}-\frac{1}{4}(\kappa x)_{a c} \partial_{\mu} \zeta^{c}\right) \times \\
& \left(\partial^{\mu} \tilde{\zeta}_{b}+\frac{1}{8}(\kappa x x)_{b} \partial^{\mu} \zeta_{0}-\frac{1}{4}(\kappa x)_{b d} \partial^{\mu} \zeta^{d}\right) \\
& -2 e^{2 \phi_{\mathrm{A}}} V^{-1}\left(\partial_{\mu} \tilde{\zeta}^{0}+x^{a} \partial_{\mu} \tilde{\zeta}_{a}+\frac{1}{24}(\kappa x x x) \partial_{\mu} \zeta_{0}-\frac{1}{8}(\kappa x x)_{a} \partial_{\mu} \zeta^{a}\right)^{2} \\
& -\frac{1}{4} e^{4 \phi_{\mathrm{A}}}\left(\partial_{\mu} \tilde{\phi}+\zeta^{i} \partial_{\mu} \tilde{\zeta}_{i}-\tilde{\zeta}^{i} \partial_{\mu} \zeta_{i}\right)^{2}
\end{aligned}
$$

where in anticipation of the map to type IIB we use $V=\frac{1}{6}(\kappa y y y)$ (although this does not correspond to the volume of type IIA).

The map of the field variables is obtained by comparing the two Lagrangians (20) and (38). First of all one learns that the dilatons of the the two theories have to be identified $\left(\phi_{\mathrm{A}}=\phi_{4}\right)$ which is in accord with the fact that mirror symmetry is already valid in string perturbation theory. Furthermore, one is led to the straightforward identification

$$
y^{a}=v^{a}, \quad x^{a}=b^{1 a} .
$$

The remaining terms can be systematically compared due to their specific dependence on $y^{a}$ and $\phi_{\mathrm{A}}$. One obtains

$$
\begin{aligned}
& \partial_{\mu} \zeta_{0}= \pm \sqrt{2} \partial_{\mu} l \\
& b^{1 a} \partial_{\mu} \zeta_{0}-\partial_{\mu} \zeta^{a}= \pm \sqrt{2}\left(l \partial_{\mu} b^{1 a}-\partial_{\mu} b^{2 a}\right) \\
& \partial_{\mu} \tilde{\zeta}^{a}+\frac{1}{8}\left(\kappa b^{1} b^{1}\right)_{a} \partial_{\mu} \zeta_{0}-\frac{1}{4}\left(\kappa b^{1}\right)_{a b} \partial_{\mu} \zeta^{b} \\
& = \pm \frac{\sqrt{2}}{4}\left(\partial_{\mu} g_{a}-\frac{1}{2} \kappa_{a b c}\left(b^{1 b} \partial_{\mu} b^{2 c}-b^{2 b} \partial_{\mu} b^{1 c}\right)\right) \\
& \partial_{\mu} \tilde{\zeta}^{0}+b^{1 a} \partial_{\mu} \tilde{\zeta}_{a}+\frac{1}{24}\left(\kappa b^{1} b^{1} b^{1}\right) \partial_{\mu} \zeta_{0}-\frac{1}{8}\left(\kappa b^{1} b^{1}\right)_{a} \partial_{\mu} \zeta^{a} \\
& = \pm \frac{\sqrt{2}}{4}\left(\partial_{\mu} h_{2}-b^{1 a} \partial_{\mu} g_{a}+\frac{1}{6} \kappa_{a b c} b^{1 a}\left(b^{1 b} \partial_{\mu} b^{2 c}-b^{2 b} \partial_{\mu} b^{1 c}\right)\right) \\
& \partial_{\mu} \tilde{\phi}-\tilde{\zeta}^{i} \partial_{\mu} \zeta_{i}+\zeta^{i} \partial_{\mu} \tilde{\zeta}_{i} \\
& = \pm\left(\partial_{\mu} h_{1}+l \partial_{\mu} h_{2}+b^{2 a} \partial_{\mu} g_{a}-l b^{1 a} \partial_{\mu} g_{a}-\frac{1}{6} \kappa_{a b c} b^{2 a}\left(b^{1 b} \partial_{\mu} b^{2 c}-b^{2 b} \partial_{\mu} b^{1 c}\right)\right. \\
& \left.\quad+\frac{1}{6} l \kappa_{a b c} b^{1 a}\left(b^{1 b} \partial_{\mu} b^{2 c}-b^{2 b} \partial_{\mu} b^{1 c}\right)\right) \cdot
\end{aligned}
$$


This system of equations is solved by

$$
\begin{aligned}
\zeta^{0} & =\sqrt{2} l \\
\zeta^{a} & =\sqrt{2}\left(l b^{1 a}-b^{2 a}\right), \\
\tilde{\zeta}_{a} & =-\frac{\sqrt{2}}{4} g_{a}+\frac{\sqrt{2}}{8} l\left(\kappa b^{1} b^{1}\right)_{a}-\frac{\sqrt{2}}{8}\left(\kappa b^{1} b^{2}\right)_{a}, \\
\tilde{\zeta}_{0} & =\frac{\sqrt{2}}{4} h_{2}-\frac{\sqrt{2}}{24} l\left(\kappa b^{1} b^{1} b^{1}\right)+\frac{\sqrt{2}}{24}\left(\kappa b^{1} b^{1} b^{2}\right), \\
\tilde{\phi} & =h_{1}+\frac{1}{2} l h_{2}+\frac{1}{2} b^{2 a} g_{a}-\frac{1}{2} l b^{1 a} g_{a}-\frac{1}{12}\left(\kappa b^{1} b^{2} b^{2}\right)+\frac{1}{12} l\left(\kappa b^{1} b^{1} b^{2}\right),
\end{aligned}
$$

which gives the desired relation between the type IIA and type IIB field variables. It is somewhat surprising that this relation is so involved. Remarkably (41) can be inverted

$$
\begin{aligned}
l & =\frac{1}{\sqrt{2}} \zeta_{0}, \\
b^{2 a} & =\frac{1}{\sqrt{2}}\left(x^{a} \zeta_{0}-\zeta^{a}\right), \\
g_{a} & =-\frac{4}{\sqrt{2}} \tilde{\zeta}_{a}+\frac{1}{2 \sqrt{2}}(\kappa x \zeta)_{a}, \\
h_{2} & =\frac{4}{\sqrt{2}} \tilde{\zeta}_{0}+\frac{1}{6 \sqrt{2}}(\kappa x x \zeta), \\
h_{1} & =\tilde{\phi}-\zeta^{i} \tilde{\zeta}_{i}+\frac{1}{6}(\kappa x \zeta \zeta)-\frac{1}{12} \zeta_{0}(\kappa x x \zeta) .
\end{aligned}
$$

\subsection{Worldsheet Instanton Corrections}

After having established the map between the type IIA and type IIB variables in the large volume/large complex structure limit we can discuss the corrections away from this limit. Mirror symmetry assures the equivalence of IIA and IIB compactified on mirror manifolds and it becomes a matter of convenience in what variables one describes the action. The $\alpha^{\prime}$ corrections to the large volume limit are most easily discussed in terms of type IIA variables and the holomorphic prepotential $f(z)$. In the large volume limit $f(z)$ is cubic (cf. (34)) while sub-leading $\left(\alpha^{\prime}\right)$ corrections are known to be [18, 22, 23]

$$
f(z)=\frac{i}{3 !} \kappa_{a b c} z^{a} z^{b} z^{c}+c \chi \zeta(3)+\sum_{d_{a}} n_{d_{a}} L i_{3}\left(e^{-2 \pi d_{a} z^{a}}\right) .
$$

Here $c$ is a normalization factor, $\chi=2\left(h_{(1,1)}-h_{(1,2)}\right)$ is the Euler number, $d_{a}$ is an $h_{(1,1)}$-dimensional summation index, $n_{d_{a}}$ are $h_{(1,1)}$ model dependent integer coefficients and

$$
L i_{3}(x)=\sum_{j=1}^{\infty} \frac{x^{j}}{j^{3}}
$$


In order to obtain the type IIB effective action (in terms of type IIA variables) one inserts (43) into (29) using (30)-(33). The same action can be expressed in terms of type IIB variables by using the transformations (39) and (41). This last step is necessary in order to discuss the fate of the $S L(2, \mathbf{Z})$ symmetry away from the large volume limit. It is this aspect we now turn to.

\subsection{Symmetry Properties}

Let us first identify the PQ symmetries of type IIB in the type IIA Lagrangian (29). It is invariant under $2 \times h_{(1,2)}+3$ continuous PQ symmetries (with parameters $\gamma_{i}, \tilde{\gamma}_{i}, \alpha$ ) which act on the fields as follows

$$
\delta \zeta_{i}=\gamma_{i}, \quad \delta \tilde{\zeta}_{i}=\tilde{\gamma}_{i}, \quad \delta \tilde{\phi}=\alpha+\tilde{\gamma}_{i} \zeta^{i}-\gamma_{i} \tilde{\zeta}^{i}
$$

The $2 \times\left(h_{(1,2)}+1\right)$ symmetries of the first two terms are due to the fact that $\zeta_{i}$ and $\tilde{\zeta}_{i}$ arise in the R-R sector while the last symmetry is the PQ symmetry which any scalar dual to an antisymmetric tensor has. In the large complex structure limit the Lagrangian (38) is invariant under $h_{(1,2)}$ additional continuous PQ symmetries which act on $x^{a}$ but also on $\zeta^{i}$ and $\tilde{\zeta}^{i}$ 24]

$$
\begin{aligned}
& \delta x^{a}=\hat{\gamma}^{a}, \quad \delta y^{a}=0, \quad \delta \zeta^{0}=0 \\
& \delta \tilde{\zeta}^{0}=-\hat{\gamma}_{a} \tilde{\zeta}^{a}, \quad \delta \zeta^{a}=\hat{\gamma}^{a} \zeta^{0}, \quad \delta \tilde{\zeta}^{a}=\frac{1}{4} \kappa_{a b c} \hat{\gamma}^{b} \zeta^{c} .
\end{aligned}
$$

The total number of $\left(3 \times h_{(1,2)}+3\right)$ PQ symmetries in the type IIA theory correspond to the $\left(3 \times h_{(1,2)}+2\right)$ PQ symmetries of type IIB displayed in (23), (24) together with one of the generators of the $S L(2, \mathbf{Z})$. More precisely, the $S L(2, \mathbf{Z})$ of eqs. (6) can be generated by

$$
\lambda \mapsto \lambda+1, \quad \lambda \mapsto-\frac{1}{\lambda}
$$

where the first transformation is nothing but the 'missing' PQ symmetry. ? $^{9}$

Worldsheet instantons break (46) to a discrete subgroup. It is a generic feature of perturbative string theory that continuous PQ symmetries of scalars arising in the NS-NS sector are broken to a discrete subgroup by strong coupling effects on the worldsheet. PO On the other hand PQ symmetries of fields in the R-R sector are protected and survive the perturbative expansion of string theory. This discussion also applies for the $S L(2, \mathbf{Z})$; the first transformation of (47) shifts $l$ of the R-R sector and is preserved in perturbation theory. The second transformation of (47) transforms the dilaton and in section 3.3 we observed that the entire (47) is a symmetry in the large volume limit. In order to discuss the fate of the symmetry away from this limit we need the transformation properties of the IIA variables in slightly more detail. Using (39), (41) (42) and (25)-(27) one derives

\footnotetext{
${ }^{8}$ Note that this symmetry is only given in its infinitesimal form.

${ }^{9}$ Since at the tree level the symmetry group is really $S L(2, \mathbf{R})$ this PQ symmetry is also continuous.

${ }_{10}$ An exception to this rule are the scalars which are dual to antisymmetric tensors of the NS-NS sector. For example the PQ symmetry of $\tilde{\phi}$ in eq. (45) is preserved in string perturbation theory.
} 
for the second generator of (47) the following transformation law of the type IIA variables

$$
\begin{aligned}
& e^{-2 \phi_{4}} \mapsto \frac{\sqrt{2} e^{-2 \phi_{4}}}{\sqrt{\zeta_{0}^{2}+2 V^{-1} e^{-2 \phi_{4}}}} \\
& y^{a} \mapsto \frac{1}{\sqrt{2}} \sqrt{\zeta_{0}^{2}+2 V^{-1} e^{-2 \phi_{4}}} y^{a}, \\
& x^{a} \mapsto-\frac{1}{\sqrt{2}}\left(x^{a} \zeta_{0}-\zeta^{a}\right), \\
& \zeta_{0} \mapsto-\frac{2 \zeta_{0}}{\left(\zeta_{0}^{2}+2 V^{-1} e^{-2 \phi_{4}}\right)}, \\
& \zeta^{a} \mapsto-\sqrt{2} x^{a}+\sqrt{2} \frac{\zeta_{0}\left(x^{a} \zeta_{0}-\zeta^{a}\right)}{\left(\zeta_{0}^{2}+2 V^{-1} e^{-2 \phi_{4}}\right)}, \\
& \tilde{\zeta}^{a} \mapsto \tilde{\zeta}^{a}-\frac{1}{4}(\kappa x \zeta)_{a}+\frac{1}{8} \zeta_{0}(\kappa x x)_{a}-\frac{\zeta_{0} \kappa_{a b c}\left(x^{b} \zeta_{0}-\zeta^{b}\right)\left(x^{c} \zeta_{0}-\zeta^{c}\right)}{8\left(\zeta_{0}^{2}+2 V^{-1} e^{-2 \phi_{4}}\right)}, \\
& \tilde{\zeta}^{0} \mapsto-\frac{\sqrt{2}}{4}\left(\tilde{\phi}-\zeta^{i} \tilde{\zeta}_{i}\right)+\frac{\sqrt{2}}{48}\left((\kappa x \zeta \zeta)-\zeta_{0}(\kappa x x \zeta)+\zeta_{0}^{2}(\kappa x x x)\right) \\
& -\frac{\sqrt{2}}{48} \frac{\zeta_{0} \kappa_{a b c}\left(x^{a} \zeta_{0}-\zeta^{a}\right)\left(x^{b} \zeta_{0}-\zeta^{b}\right)\left(x^{c} \zeta_{0}-\zeta^{c}\right)}{\left(\zeta_{0}^{2}+2 V^{-1} e^{-2 \phi_{4}}\right)} \\
& \tilde{\phi} \mapsto \sqrt{2}\left(2 \tilde{\zeta}^{0}-x^{a} \tilde{\zeta}_{a}-\frac{1}{6}(\kappa x x \zeta)+\frac{1}{24} \zeta_{0}(\kappa x x x)\right) \\
& +\frac{\zeta_{0}\left(\tilde{\phi}-\zeta^{i} \tilde{\zeta}_{i}+2\left(x^{a} \zeta_{0}-\zeta^{a}\right) \tilde{\zeta}_{a}+\frac{1}{3}(\kappa x \zeta \zeta)-\frac{1}{6} \zeta_{0}(\kappa x x \zeta)-\frac{1}{12} \zeta_{0}^{2}(\kappa x x x)\right)}{\sqrt{2}\left(\zeta_{0}^{2}+2 V^{-1} e^{-2 \phi_{4}}\right)} .
\end{aligned}
$$

Note that the Calabi-Yau moduli and the 4-dimensional dilaton mix in a complicated way and thus the complex $z^{a}=x^{a}+i y^{a}=b^{1 a}+i v^{a}$ transform not into themselves but into a linear combination involving the $\zeta^{i}$. Eqs. (48) show that the relatively simple transformations (25)-(27) become rather involved in terms of type IIA variables and as a consequence the $S L(2, \mathbf{Z})$ is hidden in the large complex structure limit of type IIA. Or in other words, the type IIA variables are not an appropriate basis to display the $S L(2, \mathbf{Z})$ symmetry.

From (48) one also learns that worldsheet instantons break the second generator of $S L(2, \mathbf{Z})$. Inserting (48) into (43) shows that the prepotential transforms into a function involving arbitrarily high powers of the dilaton $e^{-2 \phi_{4}}$ which cannot be cancelled by any other term at the string tree level. Thus strong coupling (non-perturbative) effects on the string worldsheet do not respect the full $S L(2, \mathbf{Z})$. However, it is possible that once non-perturbative effects in the 4-dimensional space-time are also taken into account [25] the $S L(2, \mathbf{Z})$ is restored. Work along these lines is in progress. 


\section{Conclusion}

In this paper we compactified ten-dimensional IIB supergravity on a Calabi-Yau threefold $Y_{3}$ with an arbitrary number of harmonic $(1,1)$-forms. In the 4-dimensional Lagrangian this leads to $h_{(1,1)}$ tensor multiplets coupled to supergravity and a double-tensor multiplet. The $S L(2, \mathbf{Z})$ symmetry of 10-dimensional type IIB theory is also a symmetry of the $d=4$ action in the large volume limit where the 10-dimensional description is a good approximation. The $S L(2, \mathbf{Z})$ acts naturally on the $d=10$ field variables and therefore mixes the 4-dimensional dilaton and the Calabi-Yau moduli in a non-trivial way.

By mirror symmetry the type IIB theory is equivalent to IIA supergravity compactified on the mirror manifold $\tilde{Y}_{3}$. We verified this equivalence in the large complex structure limit of type IIA and displayed explicitly the relation between the two sets of field variables. Via this map we were able to obtain the action of the $S L(2, \mathbf{Z})$ on the type IIA field variables. Finally, we noticed that for small Calabi-Yau manifolds the $S L(2, \mathbf{Z})$ is broken by strong coupling effects on the worldsheet (worldsheet instantons).

\section{A Appendix}

Throughout this paper we use the following notations and conventions: the signature of the ten-dimensional metric is chosen as $(-++\ldots+)$, capital Latin indices are tendimensional indices, Greek indices from the middle of the alphabet are four-dimensional space-time indices and the real coordinates of the Calabi-Yau threefold are denoted by $y^{\hat{\alpha}} ; \hat{\alpha}=1, \ldots, 6$. We also use a set of complex coordinates $\xi^{\alpha}, \bar{\xi}^{\bar{\alpha}}(\alpha, \bar{\alpha}=1,2,3)$, being defined as $\xi^{1}=\frac{y^{1}+i y^{2}}{\sqrt{2}}, \xi^{2}=\frac{y^{3}+i y^{4}}{\sqrt{2}}, \xi^{3}=\frac{y^{5}+i y^{6}}{\sqrt{2}}$ together with their complex conjugates. The conventions used for Christoffel symbols and the various curvature tensors are

$$
\begin{aligned}
\Gamma_{N P}^{M} & =\frac{1}{2} g^{M Q}\left(\partial_{P} g_{N Q}+\partial_{N} g_{P Q}-\partial_{Q} g_{N P}\right) \\
\mathcal{R}_{N P Q}^{M} & =\partial_{Q} \Gamma_{N P}^{M}-\partial_{P} \Gamma_{N Q}^{M}+\Gamma_{N P}^{S} \Gamma_{S Q}^{M}-\Gamma_{N Q}^{S} \Gamma_{S P}^{M} \\
\mathcal{R}_{M N} & =\mathcal{R}_{M P N}^{P}, \quad \mathcal{R}=g^{M N} \mathcal{R}_{M N} .
\end{aligned}
$$

The purpose of this appendix is to supply more details on the derivation of the Lagrangian given in (15). It is constructed by compactifying the $d=10$ effective theory of eq. (1) on a Calabi-Yau threefold. First of all the terms which do not involve the 4form $\mathcal{D}_{M N P Q}$ can be treated with standard methods. That is, the ten-dimensional metric $g_{M N}$ is decomposed into a 4-dimensional space-time part $g_{\mu \nu}$ and a 6-dimensional internal Calabi-Yau metric $g_{\hat{\alpha} \hat{\beta}}$. This in turn forces a decomposition of the ten-dimensional Ricci scalar $\mathcal{R}_{10}$

$$
\mathcal{R}_{10}=\mathcal{R}_{4}+g^{\mu \nu} \mathcal{R}_{\mu \hat{\alpha} \nu}^{\hat{\alpha}}+g^{\hat{\alpha} \hat{\beta}}\left(\mathcal{R}_{\hat{\alpha} \mu \hat{\beta}}^{\mu}+\mathcal{R}_{\hat{\alpha} \hat{\gamma} \hat{\beta}}^{\hat{\gamma}}\right)
$$

The Calabi-Yau metric $g_{\hat{\alpha} \hat{\beta}}$ is expanded around a background metric $g_{\hat{\alpha} \hat{\beta}}^{0}$ with small deformations $\delta g_{\hat{\alpha} \hat{\beta}}$

$$
g_{\hat{\alpha} \hat{\beta}}=g_{\hat{\alpha} \hat{\beta}}^{0}+\delta g_{\hat{\alpha} \hat{\beta}} .
$$


In $d=4$ massless scalar fields arise as the inequivalent harmonic deformations of the Calabi-Yau metric [26, 16]. More precisely one expands

$$
\begin{aligned}
\delta g_{\alpha \bar{\beta}}=\hat{v}^{a}(x) \omega_{\alpha \bar{\beta}}^{a}, & a=1, \ldots, h_{(1,1)}, \\
\delta g_{\alpha \beta}=z^{A}(x) \chi_{\alpha \beta}^{A}, & A=1, \ldots, h_{(1,2)},
\end{aligned}
$$

where $\omega_{\alpha \bar{\beta}}^{a}$ are harmonic $(1,1)$-forms while $\chi_{\alpha \beta}^{A}$ are related to harmonic $(1,2)$-forms and $\hat{v}^{a}$ and $z^{A}$ are the scalar moduli. The $z^{A}$ turn out to be members of vector multiplets and therefore will be omitted in the following discussion. Inserting (51) and (52) into (50) and keeping up to quadratic terms in $\omega^{a}$ results in 12 प

$$
\mathcal{R}_{10}=\mathcal{R}_{4}-\frac{1}{2} \partial_{\mu} \hat{v}^{a} \partial^{\mu} \hat{v}^{b} \omega_{\alpha \bar{\alpha}}^{a} \omega_{\beta \bar{\beta}}^{b} g^{0 \alpha \bar{\beta}} g^{0 \beta \bar{\alpha}}+\partial_{\mu} \hat{v}^{a} \partial^{\mu} \hat{v}^{b} \omega_{\alpha \bar{\alpha}}^{a} \omega_{\beta \bar{\beta}}^{b} g^{0 \alpha \bar{\alpha}} g^{0 \beta \bar{\beta}}
$$

The antisymmetric tensors $B_{M N}^{I}$ decompose as

$$
B_{M N}^{I}=\left(\begin{array}{cc}
B_{\mu \nu}^{I} & 0 \\
0 & B_{\hat{\alpha} \hat{\beta}}^{I}
\end{array}\right) .
$$

Since there are no harmonic $(2,0)$ forms on $Y_{3}$ the $B_{\hat{\alpha} \hat{\beta}}^{I}$ are expanded only in terms of harmonic $(1,1)$-forms according to eqs. (9). Finally, the ten-dimensional integration measure splits according to

$$
\int d^{10} x \sqrt{-g_{10}}=\int d^{4} x \sqrt{-g_{4}} \int d^{6} \xi i \sqrt{g_{6}},
$$

and the integration over the Calabi-Yau threefold can be performed using (12) and (13).

The two remaining terms in eq. (11) contain the 4 -form $\mathcal{D}_{M N P Q}$. As we discussed in section 3.1 the massless modes in $d=4$ arising from dimensional reduction of $\mathcal{D}_{M N P Q}$ are vectors $\mathcal{D}_{\mu \hat{\alpha} \hat{\beta} \hat{\gamma}}$ and tensors $\mathcal{D}_{\mu \nu \hat{\alpha} \hat{\beta}}$. Not all of them are independent but related by the self-duality condition (3). The vectors are of no concern here and so in the reduction procedure we only focus on the tensor fields. In order to proceed we make an Ansatz for the couplings of the tensor fields where the individual terms are dictated by the reduction of the last terms in (1)

$$
\begin{aligned}
\mathcal{L}= & \sqrt{-g_{10}}\left(k_{1}\left(F_{\mu \nu \rho \hat{\alpha} \hat{\beta}}\right)^{2}+k_{2} \epsilon_{I J} B_{\hat{\alpha} \hat{\beta}}^{I} H_{\mu \nu \rho}^{J} F^{\mu \nu \rho \hat{\alpha} \hat{\beta}}+k_{3} \epsilon_{I J} \epsilon_{K L} B_{\hat{\alpha} \hat{\beta}}^{I} H_{\mu \nu \rho}^{J} B^{K \hat{\alpha} \hat{\beta}} H^{L \mu \nu \rho}\right) \\
& +\epsilon^{\mu \nu \rho \sigma} \epsilon^{\hat{\alpha} \hat{\beta} \hat{\gamma} \hat{\delta} \hat{\epsilon} \hat{\phi}} \epsilon_{I J}\left(k_{4} F_{\mu \nu \rho \hat{\alpha} \hat{\beta}} B_{\hat{\gamma} \hat{\delta}}^{I} \partial_{\sigma} B_{\hat{\epsilon} \hat{\phi}}^{J}+k_{5} \epsilon_{K L} H_{\mu \nu \rho}^{I} B_{\hat{\alpha} \hat{\beta}}^{J} B_{\hat{\gamma} \hat{\delta}}^{K} \partial_{\sigma} B_{\hat{\epsilon} \hat{\phi}}^{L}\right),
\end{aligned}
$$

where

$$
\begin{aligned}
F_{\mu \nu \rho \hat{\alpha} \hat{\beta}} & \equiv \frac{1}{30}\left(\partial_{\mu} \tilde{\mathcal{D}}_{\nu \rho \hat{\alpha} \hat{\beta}}+\partial_{\rho} \tilde{\mathcal{D}}_{\mu \nu \hat{\alpha} \hat{\beta}}+\partial_{\nu} \tilde{\mathcal{D}}_{\rho \mu \hat{\alpha} \hat{\beta}}\right), \\
\tilde{\mathcal{D}}_{\mu \nu \hat{\alpha} \hat{\beta}} & \equiv \mathcal{D}_{\mu \nu \hat{\alpha} \hat{\beta}}+\frac{3}{4} \epsilon_{I J} B_{\mu \nu}^{I} B_{\hat{\alpha} \hat{\beta}}^{J},
\end{aligned}
$$

\footnotetext{
${ }^{11}$ There is a slight inconsistency in the derivation of eq. (53) in ref. [12] in that not all terms contributing at quadratic order are properly taken into account. However, this merely affects the coefficients of eq. (53). We thank M. Haack for communicating the correct formula prior to publication. A more detailed derivation of eq. (53) will be given in ref. [27.
} 
and $k_{1}, \ldots, k_{5}$ are constants to be determined. This is done by comparing the equations of motion of (56) with the dimensionally reduced 10-dimensional field equations. Since we only need to fix a few constants we simplify the task by doing this comparison in the limit $l=0, \phi=$ const. and $g_{\mu \nu}=\eta_{\mu \nu}$. For our purpose the important field equations turn out to be

$$
\partial^{P} G_{M N P}=-\frac{10}{3} i F_{M N P Q S} G^{P Q S} .
$$

The general definition of $G_{M N P}$ can be found in [13] while here we only record its components in this specific limit

$$
\begin{array}{r}
G_{\mu \nu \rho}=e^{\frac{\phi}{2}}\left(e^{-\phi} H_{\mu \nu \rho}^{1}+i H_{\mu \nu \rho}^{2}\right), \\
G_{\mu \hat{\alpha} \hat{\beta}}=\frac{1}{3} e^{\frac{\phi}{2}}\left(e^{-\phi} \partial_{\mu} B_{\hat{\alpha} \hat{\beta}}^{1}+i \partial_{\mu} B_{\hat{\alpha} \hat{\beta}}^{2}\right), \\
G_{\hat{\alpha} \mu \nu}=G_{\mu \hat{\alpha} \nu}=G_{\mu \nu \hat{\alpha}}=G_{\hat{\alpha} \hat{\beta} \hat{\gamma}}=0 .
\end{array}
$$

As a consequence (58) simplifies to

$$
\begin{aligned}
\partial^{\rho} G_{\rho \mu \nu} & =-10 i F_{\mu \nu \rho \hat{\alpha} \hat{\beta}} G^{\rho \hat{\alpha} \hat{\beta}} \\
\partial^{\mu} G_{\hat{\alpha} \hat{\beta} \mu} & =-\frac{10}{3} i\left(F_{\mu \nu \rho \hat{\alpha} \hat{\beta}} G^{\mu \nu \rho}+3 F_{\mu \hat{\alpha} \hat{\beta} \hat{\gamma} \hat{\delta}} G^{\mu \hat{\gamma} \hat{\delta}}\right) .
\end{aligned}
$$

The first equation is merely an equation for $B_{\mu \nu}^{I}$ and hence not of immediate interest for us. Using the self-duality condition $F_{\mu \hat{\alpha} \hat{\beta} \hat{\gamma} \hat{\delta}} G^{\mu \hat{\gamma} \hat{\delta}}=\frac{1}{12 \sqrt{-g}} \epsilon_{\mu \nu \rho \sigma} \epsilon_{\hat{\alpha} \hat{\beta} \hat{\gamma} \hat{\delta} \hat{\phi} \hat{\phi}} F^{\nu \rho \sigma \hat{\epsilon} \hat{\phi}} G^{\mu \hat{\gamma} \hat{\delta}}$ the second equation in (60) yields for $B_{\hat{\alpha} \hat{\beta}}^{1}$

$$
\begin{aligned}
\partial^{\mu} \partial_{\mu} B_{\hat{\alpha} \hat{\beta}}^{1}= & 10 e^{\phi}\left(F_{\mu \nu \rho \hat{\alpha} \hat{\beta}}+\frac{3}{20} \epsilon_{I J} B_{\hat{\alpha} \hat{\beta}}^{I} H_{\mu \nu \rho}^{J}\right) H^{2 \mu \nu \rho} \\
& +\frac{5}{6 \sqrt{-g}} e^{\phi} \epsilon_{\mu \nu \rho \sigma} \epsilon_{\hat{\alpha} \hat{\beta} \hat{\gamma} \hat{\delta} \hat{\epsilon} \hat{\phi}}\left(F^{\nu \rho \sigma \hat{\epsilon} \hat{\phi}}+\frac{3}{20} \epsilon_{I J} B^{I \epsilon \hat{\phi} \hat{\phi}} H^{J \nu \rho \sigma}\right) \partial^{\mu} B^{2 \hat{\gamma} \hat{\delta}}
\end{aligned}
$$

This equation has to be compared with the equation of motion for $B_{\hat{\alpha} \hat{\beta}}^{1}$ obtained from (56)

$$
\begin{aligned}
\partial^{\mu} \partial_{\mu} B_{\hat{\alpha} \hat{\beta}}^{1}= & -4 e^{\phi}\left(k_{2} F_{\mu \nu \rho \hat{\alpha} \hat{\beta}}+2 k_{3} \epsilon_{I J} B_{\hat{\alpha} \hat{\beta}}^{I} H_{\mu \nu \rho}^{J}\right) H^{2 \mu \nu \rho} \\
& -\frac{4}{\sqrt{-g}} e^{\phi} \epsilon_{\mu \nu \rho \sigma} \epsilon_{\hat{\alpha} \hat{\beta} \hat{\gamma} \hat{\delta} \hat{\epsilon} \hat{\phi}}\left(2 k_{4} F^{\mu \nu \rho \hat{\gamma} \hat{\delta}}-k_{5} \epsilon_{I J} B^{I \hat{\gamma} \hat{\delta}} H^{J \mu \nu \rho}\right) \partial^{\sigma} B^{2 \hat{\epsilon} \hat{\phi}} .
\end{aligned}
$$

Comparing (61) with (62) yields

$$
k_{1}=-\frac{25}{3}, \quad k_{2}=-\frac{5}{2}, \quad k_{3}=-\frac{3}{16}, \quad k_{4}=\frac{5}{48}, \quad k_{5}=-\frac{1}{32},
$$

where the overall normalization is determined by using the gauge invariance of eqs. (21) and (22). (The analogous equations for $B_{\hat{\alpha} \hat{\beta}}^{2}$ are consistent with the same set of coefficients.) Finally one adds (56) to the dimensionally reduced action obtained from the first three terms in (11) using (53), (54) and (55), performs a Weyl rescaling $g_{\mu \nu} \rightarrow V^{-1} g_{\mu \nu}$ and integrates over the Calabi-Yau threefold using the formulae given in section 3.1. This results in the Lagrangian (15). 


\section{Acknowledgements}

This work is supported in part by the French-German binational program PROCOPE. C.H. thanks J.L. and his group for the hospitality in Halle and H.G. thanks R. Grimm and his group for the hospitality in Marseille.

This work is additionally supported by GIF - the German-Israeli Foundation for Scientific Research. J.L. thanks S. Yankielowicz and J. Sonnenschein for the hospitality in Tel Aviv and Y. Nir for the hospitality at the Weizmann Institute.

We thank I. Antoniadis, C. Bachas, B. de Wit, M. Haack, T. Mohaupt and V. Kaplunovsky for useful conversations.

\section{References}

[1] S. Kachru and C. Vafa, Nucl. Phys. B450 (1995) 69, hep-th/9505105.

[2] S. Ferrara, J.A. Harvey, A. Strominger and C. Vafa, Phys. Lett. B361 (1995) 59, hep-th/9505162.

[3] N. Seiberg, Nucl. Phys. B303 (1988) 286.

[4] S. Cecotti, S. Ferrara and L. Girardello, Int. J. Mod. Phys. A4 (1989) 2457.

[5] N. Marcus and J.H. Schwarz, Phys. Lett. 115B (1982) 111;

J.H. Schwarz, Nucl. Phys. B226 (1983) 269;

P. Howe and P.C. West, Nucl. Phys. B238 (1984) 181.

[6] E. Cremmer and B. Julia, Nucl. Phys. B159 (1979) 141.

[7] C.M. Hull and P.K. Townsend, Nucl. Phys. B438 (1995) 109, hep-th/9410167;

E. Witten, Nucl. Phys. B443 (1995) 85, hep-th/9503124;

C.M. Hull, Phys. Lett. B357 (1995) 545, hep-th/9506194.

[8] N. Berkovits, Phys. Lett. B423 (1998) 265, hep-th/9801009.

[9] M. Bodner and A.C. Cadavid, Class. Quant. Grav. 7 (1990) 829.

[10] J. Michelson, Nucl. Phys. B495 (1997) 127, hep-th/9610151.

[11] B. de Wit and A. Van Proeyen, Nucl. Phys. B245 (1984) 89;

L. Castellani, R. D'Auria and S. Ferrara, Phys. Lett. B241 (1990) 57; Class. Quant. Grav. 7 (1990) 1767;

A. Strominger, Comm. Math. Phys. 133 (1990) 163;

for a review see, for example, A. Van Proeyen, in Proceedings of the 1995 Summer School in High-Energy Physics and Cosmology, ed. by E. Gava, A. Masiero, K.S. Narain, S. Randjbar-Daemi, Q. Shafi, World Scientific, 1997, hep-th/9512139.

[12] M. Bodner, A.C. Cadavid and S. Ferrara, Class. Quant. Grav. 8 (1991) 789. 
[13] E. Bergshoeff, C. Hull and T. Ortín, Nucl. Phys. B451 (1995) 547;

E. Bergshoeff, H.J. Boonstra and T. Ortín, Phys. Rev. D53 (1996) 7206, hepth/9508091.

[14] G. Dall'Agata, K. Lechner and D. Sorokin, Class. Quant. Grav. 14 (1997) L195; hep-th/9707044;

G. Dall'Agata, K. Lechner and M. Tonin, JHEP 9807 (1998) 17, hep-th/9806140;

G. Dall'Agata, K. Lechner and M. Tonin, hep-th/9812170.

[15] A. Strominger, Phys. Rev. Lett. 55 (1985) 2547.

[16] P. Candelas and X. de la Ossa, Nucl. Phys. B355 (1991) 455.

[17] L. Dixon, in Superstrings, Unified Theories, and Cosmology ed. G. Furlan et al., World Scientific (1988);

B. Greene and R. Plesser, Nucl. Phys. B338 (1990) 15.

[18] P. Candelas, M. Lynker and R. Schimmrigk, Nucl. Phys. B341 (1990) 383;

P. Candelas, X. de la Ossa, P. Green and L. Parkes , Nucl. Phys. B359 (1991) 21;

for a review see, for example, S. Hosono, A. Klemm and S. Theisen, hep-th/9403096

and references therein.

[19] S. Ferrara and S. Sabharwal, Class. Quant. Grav. 6 (1989) L77; Nucl. Phys. B332 (1990) 317.

[20] J. Bagger and E. Witten, Nucl. Phys. B222 (1983) 1.

[21] D. Morrison, in Mirror Symmtery II, ed. by B. Greene, S.T. Yau, International Press 1997, alg-geom/9504013.

[22] P. Candelas, X. De la Ossa, A. Font, S. Katz and D. Morrison, Nucl. Phys. B416 (1994) 481, hep-th/9308083;

P. Candelas, A. Font, S. Katz and D. Morrison, Nucl. Phys. B429 (1994) 626, hep-th/9403187.

[23] J.A. Harvey and G. Moore, Nucl. Phys. B463 (1996) 315, hep-th/9510182.

[24] B. de Wit, F. Vanderseypen and A. Van Proeyen Nucl. Phys. B400 (1993) 463;

B. de Wit and A. Van Proeyen, Phys. Lett. B252 (1990) 221; Comm. Math. Phys. 149 (1992) 307, hep-th/9112027; Int. J. Mod. Phys. D3 (1994) 31, hep-th/9310067.

[25] K. Becker, M. Becker and A. Strominger, Nucl. Phys. B456 (1995) 130, hepth/9507158;

N. Berkovits and W. Siegel, Nucl. Phys. B462 (1996) 213, hep-th/9510106;

H. Ooguri and C. Vafa, Phys. Rev. Lett. 77 (1996) 3296, hep-th/9608079;

B. Greene, D. Morrison and C. Vafa, Nucl. Phys. B481 (1996) 513, hep-th/9608039;

A. Strominger, Phys. Lett. B421 (1998) 139, hep-th/9706195;

I. Antoniadis, S. Ferrara, R. Minasian and K.S. Narain, Nucl. Phys. B507 (1997) 571, hep-th/9707013;

K. Becker and M. Becker, Nucl. Phys. B551 (1999) 102, hep-th/9901126;

H. Günther, C. Herrmann and J. Louis, hep-th/9901137 and in preparation. 
[26] P. Candelas, G. Horowitz, A. Strominger and E. Witten, Nucl. Phys. B258 (1985) 46.

[27] R. Böhm, PhD-thesis, Halle University, to appear. 